Reprod. Nutr. Dévelop., 1986, 26 (1 B), 331-332.

\title{
Effet de la nature des aliments concentrés sur les lieux de la digestion d'une ration à base d'ensilage de maïs chez la vache laitière
}

H. RULQUIN

Station de Recherches sur la Vache laitière, I.N.R.A., Saint-Gilles, 35590 L'Hermitage, France.

Summary. The effects of three different concentrates (" starch ", " fiber ", " fiber + fat ") on the digestion of a maize silage diet were tested on dairy cows. Fat incorporation had no effect on diet digestion. The use of a " starch " concentrate resulted in decreased fiber digestion in the rumen and a shift in the digestion of organic matter and starch from the rumen to the intestines.

Les aliments concentrés amylacés semblent réduire davantage l'ingestion d'ensilage de maïs et la digestibilité de la ration que des aliments concentrés plus riches en parois (Vérité et Dulphy, 1981). Nous avons cherché à expliquer ces différences en étudiant les effets du type d'aliments concentrés sur la digestion de la ration.

Matériel et méthodes. Trois types d'aliments concentrés ont été comparés: un aliment " Amidon » (30\% d'orge, $30 \%$ de maîs et $30 \%$ de tourteau de soja), un aliment "Parois " $134 \%$ de pulpes de betteraves, $37 \%$ de son de blé, $21 \%$ de tourteau de soja), et un aliment "Parois + lipides " voisin du précédent mais contenant $24 \%$ de graines de soja extrudées au lieu du tourteau. Cette comparaison a été effectuée sur des vaches Pie-noires suivant un carré latin $3 \times 3$ avec des périodes de trois semaines. La ration a été constituée (en MS) de $61 \%$ d'ensilage de maïs et de $38 \%$ d'aliment concentrés. Les teneurs (en \% de la MS) en cellulose Weende des régimes "Amidon ", "Parois ", "Parois + lipides" ont été respectivement de 12,0,14,4,14,3, celles d'amidon de 32,3, 20,5, 21,6 et celles d'extrait éthéré de $3,1,3,0,4,4$.

Au cours de la dernière semaine de chaque période nous avons déterminé, la digestibilité, la composition des contenus de duodénum et de rumen (échantillon moyen de 18 prélèvements), les flux de digesta entrant dans le duodénum (double marquage P.E.G. et $\mathrm{Cr} 203$ ) et la dégradation de l'ensilage dans le rumen (méthode " in sacco $48 \mathrm{~h}$ »).

Résultats et discussion. La digestibilité et le lieu de digestion de la matière organique (MO) et de la cellulose ont été semblables pour les régimes "Parois" et "Parois + lipides » (tabl. 1). L'utilisation de graines extrudées a sans doute permis d'éviter les effets dépressifs des lipides sur la digestion dans le rumen (Palmquist, 1984). La digestibilité de la MO du régime "Amidon " a été plus élevée $(2$ points) que celle du régime "Parois", mais, comme dans les essais de Vérité et Dulphy (1981), l'écart par rapport à la valeur attendue a été plus élevé (- 4,6 vs $-3,5$ points). La part prise par le rumen dans cette digestion a été plus faible (50 vs 61) qu'avec les régimes "Parois" (tabl. 1). 
Le surplus d'amidon apporté par le concentré " Amidon " à un fourrage déjà riche $(30,7 \% \mathrm{MS})$, peut expliquer une partie de ces différences. En effet, $25 \%$ de l'amidon ont échappé aux dégradations ruminales, ce qui représente $70 \%$ du supplément de flux duodénal de MO (tabl. 1). La baisse de l'activité "Cellulolytiques " du rumen avec le régime "Amidon", indiquée par les tests in sacco (tabl. 1), a aussi accru le flux de MO alimentaire vers I'intestin. Elle explique aussi, en partie, la diminution de la digestibilité (7 points) de la cellulose (tabl. 1). L'azote a été digéré de manière identique dans les trois régimes (tabl. 1).

TABL. 1. - Effets de la nature de l'aliment concentré sur la digestion de la ration.

\begin{tabular}{|c|c|c|c|c|c|}
\hline & & Amidon & Parois & $\begin{array}{l}\text { Parois } \\
+ \text { Lipides }\end{array}$ & E.T.M. \\
\hline $\begin{array}{l}\text { Matière } \\
\text { organique }\end{array}$ & $\begin{array}{l}\text { Ingérée }(\mathrm{kg} / \mathrm{j}) \\
\text { Passant au duodénum }(\mathrm{kg} / \mathrm{j}) \\
\text { Digestible (1) }(\%) \\
\text { Digérée dans le rumen (2) (\%) }\end{array}$ & $\begin{array}{l}17,00 \\
10,82 \\
72,2 a \\
50,1\end{array}$ & $\begin{array}{l}17,18 \\
9,93 \\
70,0 b \\
60,3\end{array}$ & $\begin{array}{l}17,16 \\
9,62 \\
69,3 b \\
63,5\end{array}$ & $\begin{array}{l}0,27 \\
0,35 \\
0,08 \\
3,31\end{array}$ \\
\hline Amidon & $\begin{array}{l}\text { Ingéré }(\mathrm{kg} / \mathrm{j}) \\
\text { Passant au duodénum }(\mathrm{kg} / \mathrm{j}) \\
\text { Digestible }(1)(\%) \\
\text { Digérée dans le rumen }(2)(\%)\end{array}$ & $\begin{array}{l}5,90 \mathrm{a} \\
1,65 \mathrm{a} \\
96,0 \mathrm{a} \\
75,2 \mathrm{a}\end{array}$ & $\begin{array}{l}3,77 \mathrm{~b} \\
1,01 \mathrm{~b} \\
93,4 \mathrm{~b} \\
78,9 \mathrm{ab}\end{array}$ & $\begin{array}{l}4,01 \mathrm{~b} \\
0,75 \mathrm{~b} \\
92,8 \mathrm{~b} \\
87,8 \mathrm{~b}\end{array}$ & $\begin{array}{l}0,16 \\
0,04 \\
0,07 \\
1,29\end{array}$ \\
\hline $\begin{array}{l}\text { Cellulose } \\
\text { Weende }\end{array}$ & $\begin{array}{l}\text { Ingérée }(\mathrm{kg} / \mathrm{j}) \\
\text { Digestible }(\%) \\
\text { Digestion du fourrage in sacco (3) }(\%)\end{array}$ & $\begin{array}{l}2,21 a \\
45,7 a \\
65,2\end{array}$ & $\begin{array}{l}2,68 \mathrm{~b} \\
53,6 \mathrm{~b} \\
68,9\end{array}$ & $\begin{array}{l}2,66 b \\
51,7 b \\
68,3\end{array}$ & $\begin{array}{l}0,05 \\
0,48 \\
0,65\end{array}$ \\
\hline Azote & $\begin{array}{l}\text { Ingéré }(\mathrm{g} / \mathrm{j}) \\
\text { Passant au duodénum (NNA g/j) } \\
\text { Digestible (\%) } \\
\text { Ammoniaque du jus de rumen (4) (mg//) }\end{array}$ & $\begin{array}{l}449 \\
455 \\
68,5 \\
143\end{array}$ & $\begin{array}{c}432 \\
469 \\
67,3 \\
109\end{array}$ & $\begin{array}{l}436 \\
446 \\
68,0 \\
134\end{array}$ & $\begin{array}{r}8,05 \\
10,95 \\
0,61 \\
17,00\end{array}$ \\
\hline
\end{tabular}


non ammoniacal ; (3) MS perdue en $48 \mathrm{~h} ;\left(^{4}\right)$ teneur moyenne sur $24 \mathrm{~h}$. Les valeurs $n^{\prime}$ ayant pas les mêmes lettres différent à $\mathrm{P}<0,05$.

La diminution de l'activité cellulolytique des microorganismes du rumen et le report de la digestion du rumen vers l'intestin grêle enregistrés avec l'aliment concentré amylacé amènent à penser que, l'origine du pouvoir d'encombrement élevé (Vérité et Dulphy, 1981) de ces aliments est digestive.

Palmquist D. L., 1984. Uses of fats in diets for lactating dairy cows, p. 357-381. In Wiseman J., Fats in animal nutrition, Butterworths.

Vérité R., Dulphy J. P., 1981. Effet de la nature de l'aliment concentré sur l'ingestion et les performances des vaches laitières. Bull. techn. C.R.Z.V. Theix, I.N.R.A., 45, 15-21. 